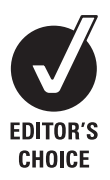

1 Department of Internal

Medicine, Kaohsiung Veterans General Hospital, Kaohsiung,

Taiwan, Republic of China;

${ }^{2}$ School of Medicine, National Yang-Ming University, Taipei, Taiwan, Republic of China;

${ }^{3}$ Department of Nuclear

Medicine, Kaohsiung Veterans General Hospital, Kaohsiung,

Taiwan, Republic of China;

${ }^{4}$ Department of Radiology,

Kaohsiung Veterans General

Hospital, Kaohsiung, Taiwan,

Republic of China

Correspondence to: Dr Ming-Ting Wu, Section of Thoracic and Circulation Imaging, Department of Radiology; Kaohsiung Veterans General Hospital, No 386, TaChung 1st Road, Kaohsiung, Taiwan 813, Republic of China: wu.mingting@gmail.com

Accepted 12 May 2009 Published Online First

3 June 2009

\title{
Dual-phase multi-detector computed tomography assesses jeopardised and infarcted myocardium subtending infarct-related artery early after acute myocardial infarction
}

\author{
K-R Chiou, ${ }^{1,2}$ W-C Huang, ${ }^{1,2}$ N-J Peng, ${ }^{2,3}$ Y-L Huang, ${ }^{2,4}$ S-H Hsiao, ${ }^{1,2}$ K-H Chen, ${ }^{2,4}$ \\ $\mathrm{M}-\mathrm{T} \mathrm{Wu}^{2,4}$
}

\section{ABSTRACT}

Objectives: To investigate dual-phase multi-detector computed tomography (MDCT) for assessing extent and severity of jeopardised and infarcted myocardium subtended by infarct-related artery (IRA), and its indication for revascularisation after acute myocardial infarction (AMI).

Designs, setting and patients: Prospective, singlecentre study included 107 patients with uncomplicated post-AMI 3-7 days, who met criteria and underwent dual-phase 64-slice MDCT. IRA, culprit lesion and extent of jeopardised/infarcted myocardium were assessed by three-dimensional (3D) volume-rendered images with myocardium maps and computed tomography angiography (CTA), compared with stress-redistribution thallium201 single-photon emission computed tomography (SPECT) plus conventional coronary angiography (CCA). MDCT-jeopardised score (severity of jeopardised myocardium) was defined as extent of jeopardised myocardium multiplied by the weighted factor dependent on culprit lesion severity compared with SPECT-SRS (summation of segmental reversible score). The IRA indication for revascularisation was evaluated by MDCT-jeopardised score plus CTA. SPECT-SRS $\geqslant 2$ plus CCA-culprit lesion $\geqslant 50 \%$ was the standard reference.

Results: The presence of MDCT-delayed enhancement was found in 101 (94.4\%) patients. The IRA and culprit lesion were identified in 99 (92.5\%) patients by MDCTmyocardium maps plus CTA. The concordance between MDCT and SPECT for detecting infarcted myocardium was good (kappa $=0.702$ ). The correlation between MDCTjeopardised score and SPECT-SRS was 0.741. The correlation between CTA and CCA for culprit lesion severity was 0.85 . The sensitivity, specificity, negative and positive predictive values of MDCT-jeopardised score $\geqslant 2.5$ plus CTA for indicating revascularisation were $90.2 \%, 80.4 \%, 86.0 \%$ and $85.9 \%$, respectively.

Conclusions: Dual-phase MDCT has good accuracy for identifying IRA, and assessing infarcted and jeopardised myocardium for clinical relevance. It provides an alternative for triage and therapeutic planning in postAMl.

Appropriate risk triage and therapeutic decisionmaking in patients with acute myocardial infarction (AMI) who have not undergone primary or rescue angioplasty requires information about the status of infarct-related territory regarding the area of irreversibly injured myocardium (infarcted myocardium) and the area at risk for post-infarct ischaemia (jeopardised myocardium). ${ }^{12}$ Currently, single-photon emission computed tomography (SPECT) is widely accepted as the reference standard in physiologically recognising jeopardised and infarcted myocardium. If revascularisation is necessary, invasive conventional coronary angiography (CCA) is added for assigning the infarct-related artery (IRA) and evaluating culprit lesion severity.

Technological advances in multi-detector computed tomography (MDCT) provide computed tomography angiography (CTA) with high accuracy in detecting significant coronary artery disease in comparison with $\mathrm{CCA}^{3-5}$ and robust quality for evaluating left ventricular (LV) function. ${ }^{6}$ Furthermore, infarcted myocardium can be identified and quantified by MDCT late phase delayed enhancement (DE) based on differential wash-in and washout kinetics of the contrast agent..$^{7-11}$ Accordingly, we hypothesised that dual-phase 64 slice MDCT could specifically assess the extent of jeopardised and infarcted myocardium subtended by infarct-related artery (IRA) and determine its indication for revascularisation early post-AMI, compared with stress-redistribution thallium-201 (T1-201) SPECT plus CCA.

\section{PATIENTS AND METHODS}

\section{Study design and patient population}

From September 2007 to August 2008, AMI patients admitted to our cardiovascular centre who did not receive primary or rescue angioplasty were evaluated to participate in this prospective study. Diagnosis of myocardial infarction (MI) was defined by typical angina lasting $\geqslant 30$ minutes; biochemical evidence of peak creatine phosphokinase over twice the normal upper limit and/or new electrocardiographic (ECG) changes including development of $\mathrm{Q}$ wave or ST/T changes lasting 48 hours or longer. Patients were excluded if they had history of previous MI, resting angina beyond the initial 24 hours, developing functional class III or IV heart failure, cardiomyopathy, contraindication to iodated contrast agent, atrial fibrillation or serum creatinine $>1.6 \mathrm{mg} / \mathrm{dl}$. Figure 1 shows the flowchart of the study design and lists for patient ineligibility. Patients underwent MDCT and stressredistribution SPECT protocol 3-7 days after AMI, followed by CCA within 1-2 days using standard techniques. The study was approved by our institutional review board; all participants gave written informed consent. 
Figure 1 Flowchart of the study design. Excluded patients are shown in dotted boxes. The median time (range) from acute myocardial infarction (AMI) is shown on the left. CPK, creatine phosphokinase; Echo, echocardiography; LV, left ventricular; NYHF, New York Heart Association functional class; SPECT, single-photon emission computed tomography.

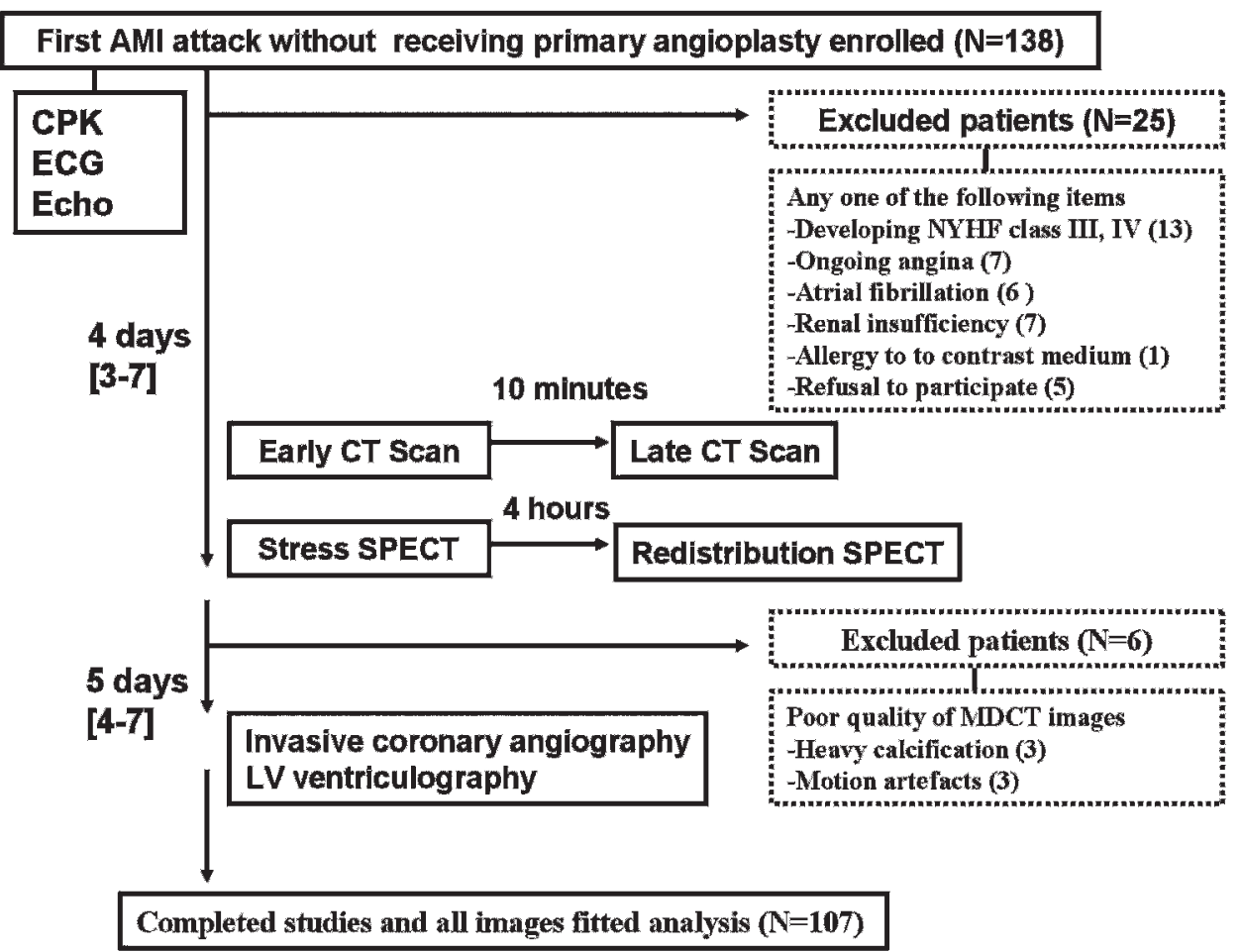

\section{MDCT protocol}

MDCT examination was performed with Toshiba Multi-Slice Aquilion 64 system (Toshiba Medical Systems Corporation, Otawara, Japan). $\beta$-Blockers were administered one hour before MDCT scans if heart rates were $>60 / \mathrm{min}$. Nitrolingual spray (2 puffs, $0.4 \mathrm{mg} /$ dose; Pohl-Boskamp GmBH, Hohenlockstedt, Germany) was administered 2 minutes before scanning. For early phase images, scan parameters were $64 \times 0.5-\mathrm{mm}$ collimation, rotation time $350 \mathrm{~ms}$, pitch factor $0.2-0.3$, tube voltage $120 \mathrm{kV}$, tube current $400 \mathrm{~mA}$ (range 300-600) and ECGtriggered dose modulation. Contrast agent (iopromide; $350 \mathrm{mg} / \mathrm{ml}$, Schering, Berlin, Germany) was injected at a flow rate of $4-5 \mathrm{ml} / \mathrm{s}$ (volume dose, $1.0 \mathrm{ml} / \mathrm{kg}$ body weight), followed by $30 \mathrm{ml}$ saline solution flush, via a power injector system. Once signal intensity at the ascending aorta reached the predefined threshold of 150 Hounsfield units, the helical scan started automatically. Images were acquired during a single breath hold. After early phase acquisition, additional iopromide $(0.4 \mathrm{ml} / \mathrm{kg})$ was infused at a rate of $0.50 \mathrm{ml} / \mathrm{s}$. Ten minutes later, late phase images were acquired. Detector collimation was increased to $1.5 \mathrm{~mm}$, and tube voltage was reduced to 80-100 kV depending on patient's body size. The effective radiation dose was estimated from the product of dose-length product times a conversion coefficient for the chest $\left(\mathrm{k}=0.017 \mathrm{mSv} \cdot \mathrm{mGy}^{-1} \cdot \mathrm{cm}^{-1}\right){ }^{12}$ Early phase dose was 10 $15 \mathrm{mSv}$ and late phase was 3-5 mSv. Raw data from the scans were reconstructed using a half-scan algorithm for patients with heart rate $<65$ beats/min and segmental (two or three) reconstruction algorithm for heart rate $>65$ beats $/ \mathrm{min}$.

\section{Stress-redistribution TI-201 SPECT data acquisition}

Dipyridamole $0.56 \mathrm{mg} / \mathrm{kg}$ body weight was infused intravenously for 4 minutes with patients in a supine position. Four minutes after discontinuation, patients received $111 \mathrm{MBq}$ of thallium-201 (Tl-201) intravenously. Imaging was performed using Siemens Symbia-T SPECT-CT imaging systems (Siemens
Medical Solutions, Hoffman Estates, IL, USA) at 5-10 minutes and 4 hours after Tl-201 injection. Acquisition was synchronised with ECG with an acceptance window of $20 \%$; each projection was divided into eight images per cardiac cycle. Images were acquired in a $64 \times 64$ matrix with a 1.50 acquisition zoom and reconstructed by filtered back projection with Butterworth filter with an order of 5 and critical frequency of 0.45 cycles per pixel.

\section{MDCT image analysis}

MDCT scans were evaluated using a dedicated workstation (Vitrea 2; Vital Images, Minnetonka, MI, USA). All scans were interpreted separately by two experienced reviewers blinded to patient data and SPECT results. Three steps were performed, as shown in figure $2 \mathrm{~A}$ and $\mathrm{B}$.

Step 1 for location of infarcted myocardium

Step 1 for location of infarcted myocardium was evaluated. ${ }^{11}$ Late phase MDCT scans were reconstructed by multiplanar reformation (MPR) and transformed into short-axis, transverseaxis and long-axis views, which were simultaneously displayed on the workstation (window-width/level 120-160/80-120 HU). The LV myocardium, divided into 17 segments as proposed by the American Heart Association, ${ }^{13}$ was examined for the presence of hyperenhanced regions, defined as increased signal regions with a mean difference of at least 20 Hounsfield units to differentiate infarcted from normal myocardium, measured by using regions of interest of about $20 \mathrm{~mm}^{2}$ within both hyperenhanced and contiguous normal regions. Subendocardial areas of hypoenhancement within the hyperenhanced area were also considered part of the hyperenhanced area.

Step 2 assessed coronary plaque severity

Step 2 assessed coronary plaque severity (lesion stenosis and length). Following inspection of $3 \mathrm{D}$ volume-rendered images, 
Figure 2 MDCT image analysis in a 50 year-old-man post-AMI (4 days) compared with SPECT plus conventional coronary angiography (CCA). (A) Shortaxis views show delayed enhancement (DE) in the inferior-lateral wall. (B) 3D inverse angio-emulation view (left upper) and curved multiplanar reformation image (left lower) show right coronary artery (RCA) having total occlusion with contrast filling distal to occlusion (arrows); left anterior descending artery and left circumflex artery are normal. Sketches illustrate how to assess extent of infarcted/jeopardised myocardium, under a3D volume-rendered image from posterior view by a 17 -segment model. Extent of infarcted/jeopardised myocardium was 5 segments (S4-5, S9$10, S 15), 35.4 \%$ area was infarcted myocardium (DE was planimetered and calculated in short-axis images with 8$\mathrm{mm}$ slice thickness along long-axis). The extent of jeopardised myocardium is 3.23 $(=5 \times(100 \%-35.4 \%))$ and jeopardised score is 6.46. (C) SPECT images show partial reversible perfusion in S4-5, S1011 and S15; summated reversible score is 5. (D) CCA shows RCA having total occlusion (arrow) and collateral from left coronary artery.
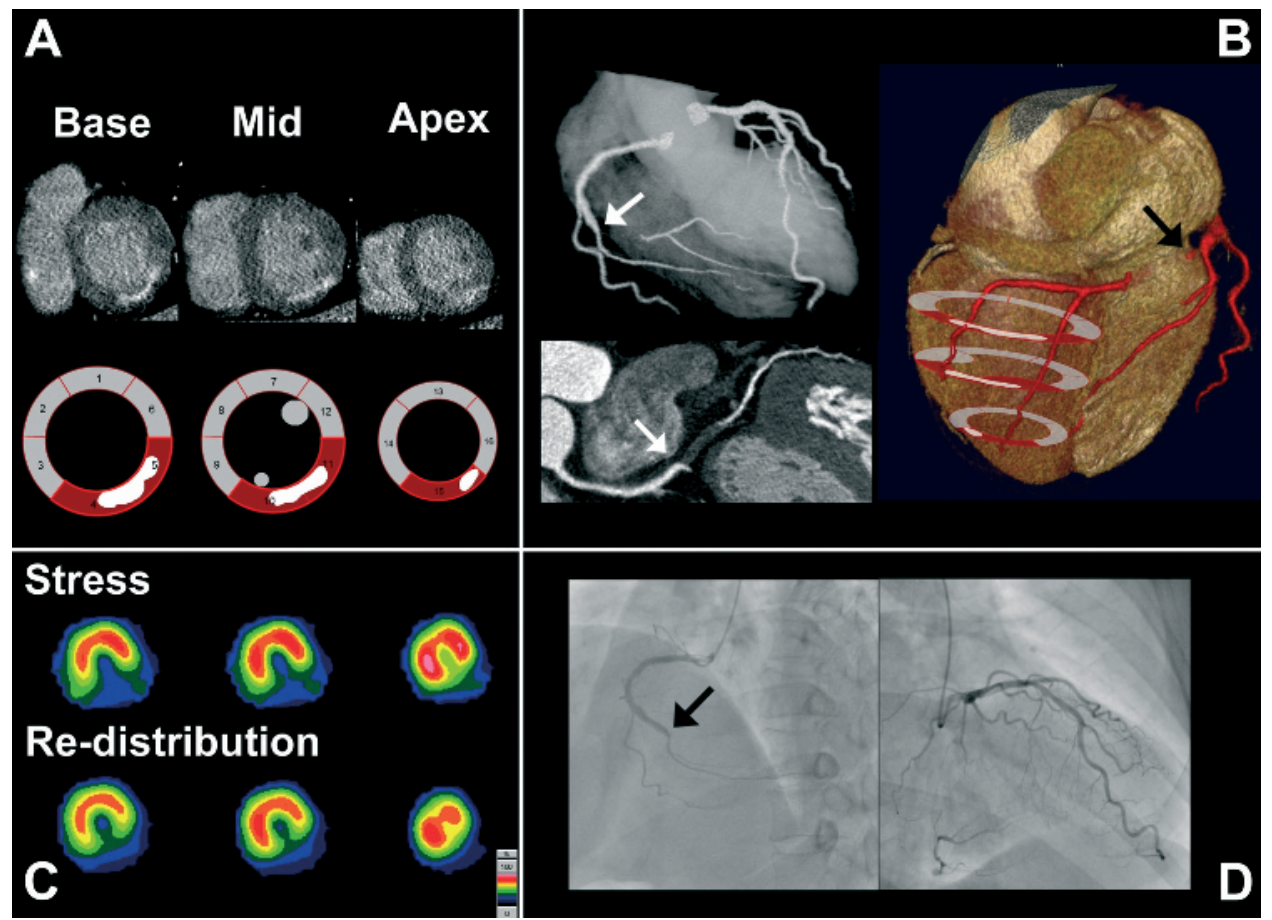

the coronary arteries variation, including vessel dominance (right, left or co-dominance), and left anterior descending artery termination (before LV apex, reaching apex but not supplying the inferoapical segment, or wrapping around apex and supplying the inferoapical segment) ${ }^{14}$ were categorised. Coronary plaques were evaluated on the basis of axis images, curved multiplanar reconstruction images, cross-sectional images perpendicular to the vessel centre line (1-mm thickness) and were quantified at the site of maximal lumen narrowing and reference sites proximal and distal to the lesion. ${ }^{15}$

Step 3 for identification of IRA, culprit lesion and jeopardised/ infarcted myocardium

IRA subtending segments with presence of DE were ascertained by $3 \mathrm{D}$ volume-rendered images with CTA gross anatomy. If there were multiple plaques in IRA, the lesion with lower density and larger plaque area was considered as the MDCT culprit lesion. ${ }^{15}$ Jeopardised/infarcted myocardium was assessed by $3 \mathrm{D}$ rendered images (using $3 \mathrm{D}$ volume with Vessel Probe (Vital Images), 3D angio-emulation view and transparent background rendering of myocardium), which simultaneously displayed LV myocardium, culprit lesion and dominant and major side-branches of the IRA and were used to identify the number of segments supplied by the culprit lesion.

From the three steps mentioned, we further measured four infarct-related parameters as follows:

1. Extent of jeopardised/infarcted myocardium, which was defined as the total number of myocardial segments, semiquantified by a 17 -segment model, ${ }^{13}$ subtending distal to the culprit lesion of IRA.

2. Extent of infarcted myocardium, which was defined as the total area of DE. Short-axis images (slice thickness $8 \mathrm{~mm}$, slice distance $8 \mathrm{~mm}$ from LV outflow tract to apex on long-axis length) were used to measure DE. DE-segmental extent was manually planimetered, calculated, divided by total area of extent of jeopardised/infarcted myocardium and expressed as a percentage.

3. Extent of jeopardised myocardium, which was defined as extent of jeopardised/infarcted myocardium minus the total area with the presence of DE.

4. Jeopardised score, representing severity of jeopardised myocardium, was defined as extent of jeopardised myocardium multiplied by the weighted factor. The weighted factor is dependent on stenotic severity of culprit lesion ${ }^{16}$ (culprit lesion $<40 \%, 0 ; 40-70 \%, 0.5 ;>70-90 \%, 1 ;>90 \%$ stenosis with the presence of contrast filling distal to the lesion, 2 ; and $>90 \%$ stenosis with absence of contrast filling distal to the lesion, 1).

\section{SPECT image analysis}

Gated SPECT images of stress and redistribution studies were displayed in short-axis, long-axis and transverse-axis views and judged, in consensus, by two experienced observers blind to MDCT findings. The myocardium was divided into a 17segment model..$^{13}$ The myocardial region of interest with the maximum count was used as the normal reference region. Mean T1-201 activity in each segment was quantified as a proportion of maximum counts in the reference region on the image. The presence of infarcted myocardium by SPECT was defined as segments with tracer activity $<75 \%$ of maximum tracer activity on redistribution images. Allocation of IRA territory was determined by segments having a presence of infarcted myocardium attributed to major coronary artery distribution. In watershed regions, allocation was determined by overlapping the maximum contour of the defect distribution. ${ }^{17} 18$ The dominance of coronary artery and its distribution by invasive CCA findings were taken into consideration. The segments were considered irreversible if segmental Tl-201 activity was 
unchanged or increased $<10 \%$ on redistribution, compared with stress images. ${ }^{19}$ The segmental reversible score was defined as 1 if relative Tl-201 activity increased by $\geqslant 10 \%-20 \%, 2$ if $>20 \%-$ $40 \%$ and 3 if $>40 \%$ in the redistribution images. Summation of reversible score (SRS) in the IRA territory represented severity of jeopardised myocardium by SPECT; SRS $\geqslant 2$ was considered the threshold for indicating for revascularisation.

\section{CCA analysis}

Coronary angiograms were analysed by two physicians blind to MDCT data. Quantitative coronary angiography was used to measure lesions with the CAAS II system (Pie Medical, Maastricht, The Netherlands). Culprit lesions by CCA criteria were determined as follows: an intraluminal filling defect consistent with thrombus, an abrupt vessel cut-off with persistence of contrast, plaque ulceration, plaque irregularity. ${ }^{20}$ The reference standard for the IRA/culprit lesion was based on a combination of acute ECG changes, wall-motion abnormalities on contrast ventriculography and CCA findings (coronary anatomy, lesion morphology). In addition, vessel dominance, site of the left anterior descending coronary artery (LAD) termination and infarct-related segments subtending distal to the culprit lesion were also assessed.

\section{Statistical analysis}

Data are presented as number (\%) or mean (SD). Categorical data were compared using $\chi^{2}$. Continuous data were compared using the Student $t$ test. Agreement was calculated by kappa statistics. Comparison between MDCT-jeopardised score and SPECT-SRS was performed using Pearson's regression analysis. Receiver-operating characteristic (ROC) analysis evaluated optimal cut-off value of MDCT-jeopardised score; combined with CTA, it predicted diagnostic accuracy in detecting SRS $\geqslant 2$. Interobserver difference for MDCT measurements was evaluated by coefficient of variance, defined as standard deviation of absolute difference between two measurements divided by their average. A p value $<0.05$ was considered statistically significant. All calculations were performed using SPSS version 12.0.

\section{RESULTS}

\section{Patient characteristics}

In total, 113 patients meeting the criteria had MDCT, SPECT and CCA performed 3-7 days post-AMI. Mean (SD) heart rate was 62 (6) beats/min (range 46-72) during MDCT acquisition. Six patients were excluded owing to poor MDCT image quality (motion artefacts and/or heavy calcification). Thus, 107 patients with a mean (SD) age of 58 (12) years were analysed. Among 64 patients having ST-segment elevation on arrival ECG., 28 (26\%) received thrombolytic therapy within 12 hours after AMI attack; 36 did not, because of arrival delay. Sixty-six (62\%) developed Q-wave MI after admission. Demographic characteristics are shown in table 1.

\section{Dual-phase MDCT findings}

In all, 183 coronary plaques with $\geqslant 50 \%$ diameter stenosis were identified by CTA alone. The presence of MDCT-DE was found in 101 (94.4\%) patients. MDCT-myocardium maps plus CTA analysis accurately identified IRA and culprit lesion in 99 (92.5\%) patients; additional CCA data confirmed culprit lesions in the remaining eight (six patients with absence of DE, two with double lesions showing similar plaque area and low density in the IRA). MDCT results are summarised in table 2.
Table 1 Clinical characteristics of patients $(n=107)$

\begin{tabular}{|c|c|}
\hline Age (years) & $58(12)$ \\
\hline Male sex & $82(77)$ \\
\hline Hypertension & $58(54)$ \\
\hline Diabetes mellitus & $49(46)$ \\
\hline Hypercholesterolaemia & $36(34)$ \\
\hline Body mass index $\left(\mathrm{kg} / \mathrm{m}^{2}\right)$ & $25.9(3.7)$ \\
\hline Current smoker & $67(63)$ \\
\hline Previous revascularisation & $11(10)$ \\
\hline Peak creatine phosphokinase level (U/I) & $1899(2025)$ \\
\hline \multicolumn{2}{|l|}{ ECG changes } \\
\hline ST-segment elevation on arrival & $64(60)$ \\
\hline Anterior/septum wall (ECG leads V1-5) & $35(33)$ \\
\hline Inferior wall (ECG leads II, III, aVF) & $21(20)$ \\
\hline Lateral wall (ECG leads I, V5-6, aVL) & $18(17)$ \\
\hline Non-ST-segment elevation on arrival & $43(40)$ \\
\hline Q-wave development after admission & $66(62)$ \\
\hline Thrombolytic agents & $28(26)$ \\
\hline Killip classification & $1.9(0.8)$ \\
\hline Baseline heart rate (beat/min) one day before MDCT & $73(10)$ \\
\hline Heart rate (beat/min) during MDCT acquisition & $62(6)$ \\
\hline Left ventricular ejection fraction (\%) & $48(9)$ \\
\hline
\end{tabular}

The mean (SD) stenosis and length of culprit lesions were $82 \%(18 \%)$ and $16.1 \mathrm{~mm}(10.2 \mathrm{~mm})$, respectively. Twenty-nine culprit lesions were totally occluded, including 20 with the presence of contrast filling distal to occlusion, and nine without. The average of extent of jeopardised/infarcted myocardium per patient was 6.2 segments, with $36.7 \%$ of the area infarcted myocardium and $63.3 \%$ jeopardised myocardium.

The coefficient of variation of interobserver measurements for measuring the number of jeopardised/infarcted myocardium was $4.8 \%$, extent of MDCT-DE $6.9 \%$, luminal stenosis of culprit lesion $4.1 \%$ and lesion length $4.6 \%$.

\section{CCA and SPECT findings}

In total, 179 lesions with $\geqslant 50 \%$ diameter stenosis were identified by CCA. Culprit lesions in 74 (69.2\%) patients were identifiable by CCA alone using lesion morphology criteria; the remaining 33 patients, having either absence of active lesion morphology or multiple vessel disease with multiple active lesions, were confirmed by acute ECG changes and wall-motion abnormality by ventriculography. The total occlusion of a culprit lesion was identified in 27 patients, including 19 with major epicardial coronary arterial collaterals, and eight without. CCA findings and comparisons with CTA are shown in table 2.

The presence of perfusion defect ( $<75 \%$ tracer uptake) on redistribution images was identified in 97 (90.7\%) patients, distributed in 580 infarcted segments. The presence of stress reversible defect with $S R S \geqslant 2$ in the IRA territory was found in 64 patients; the median SRS was 4 (range 0-23).

\section{Comparing dual-phase MDCT and SPECT for assessment of jeopardised/infarcted myocardium subtended by IRA territory} Concordance between MDCT-DE and SPECT for detecting the presence or absence of infarcted myocardium by per-segment analysis was good (kappa value 0.702). In all, 663 jeopardised/ infarcted myocardial segments were identified by MDCT; as DE-segmental extent $(0 \%, 1-25 \%, 26-50 \%, 51-75 \%$ and $>75 \%)$ increased, the corresponding T1-201 uptake significantly decreased on redistribution images $(p<0.001)$ (fig $3 A$ ). 
Table 2 Dual-phase MDCT and CCA results and comparisons $(n=107)$

\begin{tabular}{|c|c|c|c|}
\hline & MDCT & CCA & \\
\hline \multicolumn{4}{|l|}{ Coronary artery anatomy } \\
\hline $\begin{array}{l}\text { Coronary dominance } \\
\text { right/left/co-dominance }\end{array}$ & $71 / 12 / 24$ & $71 / 12 / 24$ & $\kappa=0.930$ \\
\hline LAD terminating & & & $\kappa=0.930$ \\
\hline $\begin{array}{l}\text { Before apex/reaching apex/ } \\
\text { wrappi ng around apex and } \\
\text { supplying inferiorapical } \\
\text { segment }\end{array}$ & $11 / 42 / 54$ & $11 / 43 / 52$ & \\
\hline Number of diseased vessel & & & $\kappa=0.801$ \\
\hline Single-vessel disease & 66 & 67 & \\
\hline Two-vessel disease & 29 & 30 & \\
\hline Three-vessel disease & 12 & 10 & \\
\hline IRA/culprit lesion site* & & & * \\
\hline LM & 1 & 1 & \\
\hline $\begin{array}{l}\text { LAD: proximal/middle/distal/ } \\
\text { diagonal }\end{array}$ & $19 / 25 / 3 / 2$ & $19 / 25 / 3 / 2$ & \\
\hline $\begin{array}{l}\text { LCX: proximal/middle/distal/ } \\
\text { obtuse marginal }\end{array}$ & 6/10/8/3 & 6/10/8/3 & \\
\hline RCA: Proximal/middle/distal & $7 / 16 / 7$ & $7 / 16 / 7$ & \\
\hline \multicolumn{4}{|l|}{ Culprit lesion measurements } \\
\hline Residual diameter stenosis (\%) & $82(18)$ & $80(20.3)$ & $r=0.85$ \\
\hline $\begin{array}{l}<40 \% / 40-70 \% />70-90 \% / \\
>90-99 \% / 100 \%\end{array}$ & $8 / 20 / 27 / 23 / 29$ & 8/23/26/23/27 & \\
\hline Plaque length (mm) & $16.1(10.2)$ & $14.8(11.1)$ & $r=0.81$ \\
\hline Plaque area $\left(\mathrm{mm}^{2}\right)$ & $23.8(7.2)$ & & \\
\hline Plaque density (HU) & $36.5(14.2)$ & & \\
\hline $\begin{array}{l}\text { Extent of jeopardised/infarcted } \\
\text { segments }\end{array}$ & $6.2(2.2)$ & $6.1(2.2)$ & $r=0.95$ \\
\hline $\begin{array}{l}\text { Extent of infarcted myocardium } \\
(\%)\end{array}$ & $36.7(32.7)$ & & \\
\hline $\begin{array}{l}\text { Extent of jeopardised } \\
\text { myocardium (\%) }\end{array}$ & $63.3(34.8)$ & & \\
\hline
\end{tabular}

Data are presented as number or mean (SD). Agreement was presented by kappa value $(\kappa)$. Correlation was presented by correlation coefficient $(r)$.

*IRA/culprit lesion site: see text regarding if MDCT or CCA alone was insufficiency to identify it, how to confirm them by other modalities.

$\mathrm{CCA}$, conventional coronary angiography; $\mathrm{DE}$, delayed enhancement; $\mathrm{HU}$, Hounsfield unit; $L A D$, left anterior descending coronary artery; LCX, left circumflex; LM, left main coronary artery ; MDCT, multi-detector computed tomography; RCA, right coronary artery.

Figure 3B shows that correlation between MDCT-jeopardised score and SPECT-SRS was $0.741(p<0.001)$. Figure 4 shows ROC analysis for the extent and severity of jeopardised myocardium by our study methodology in detecting SRS $\geqslant 2$ the areas under the ROC curve (AUC) were 0.691 ( $95 \%$ CI 0.60 to 0.78 ) and 0.877 ( $95 \%$ CI 0.80 to 0.93 ), respectively. The optimal cut-off value of MDCT-jeopardised score was 2.5. If the weighted factor of culprit severity was simply classified as $<40 \%, 40-70 \%$ and $>70 \%$ and then multiplied by extent of jeopardised myocardium, AUC was 0.822 (fig 4, JPS1 curve).

\section{Accuracy of CTA plus jeopardised score to indicate IRA revascularisation}

The correlation regarding the severity of luminal stenosis of culprit lesions between CTA and CCA was 0.85 . The accuracy for CTA alone in detecting the indication for IRA revascularisation was moderate (table 3). However, the accuracy for CTA plus MDCT-jeopardised score $\geqslant 2.5$ (by ROC analysis, fig 4) was good. Compared to SPECT-SRS $\geqslant 2$ plus CCA-culprit lesion $\geqslant 50 \%$ as standard reference, the corresponding values for sensitivity, specificity, negative and positive predictive values were $90.2 \%, 80.4 \%, 86.0 \%$ and $85.9 \%$, respectively (table 3 ).
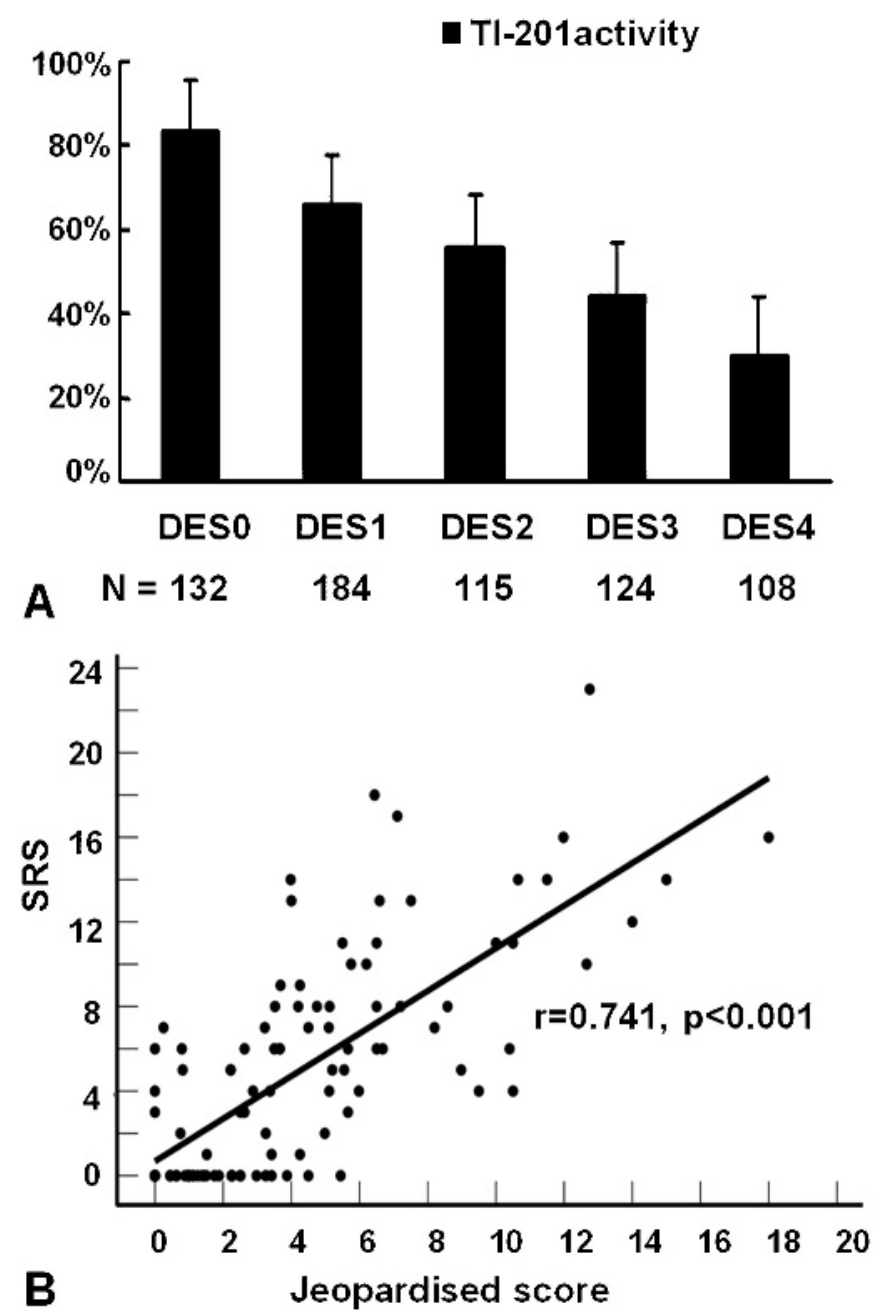

Figure 3 (A) Relation between segmental extent of delayed enhancement (DE) and thallium-201 (TI-201) activity on redistribution images in 663 jeopardised/infarcted myocardial segments. TI-201 activity decreased significantly with increasing DE extent $(p<0.001)$. DE0, 1-25\%; DE1 26-50\%; DE2 51-75\%; and DE3, >75\%. (B) Graph shows correlation between MDCT-jeopardised score and SPECT-SRS (summation of reversible score) $(r=0.741, p<0.001)$.

\section{DISCUSSION}

The prospective study focused on dual-phase MDCT-myocardium maps plus CTA to assess IRA culprit lesions and their subtending myocardium, both major determinants for guiding revascularisation and/or treatment. From these uncomplicated patients after first AMI, approximately $78 \%$ met MDCT criteria.

3D MDCT volume rendered image-guided gantry positioning system can simulate all possible angiographic views of the coronary vascular tree and LV myocardium. MDCT late-phase and early-phase images were analysed step by step, providing a high diagnostic performance (92.5\%) for assessing IRA and culprit lesions, compared with SPECT plus CCA as standard reference. The relatively low performance for CCA alone for assigning IRA can be explained as follows: (1) 41 patients (38.3\%) had non-O MI, (2) CCA was performed 4-7 days postAMI; culprit lesions would be missed by CCA criteria.

Invasive CCA can estimate the volume of myocardium subtended by a lesion if lesion location, vessel size and its dependent vascular bed are taken into consideration. ${ }^{21} 22$ Currently, MDCT-derived 3D volumetric and anatomical representations can be a non-invasive alternative. Faber et al 


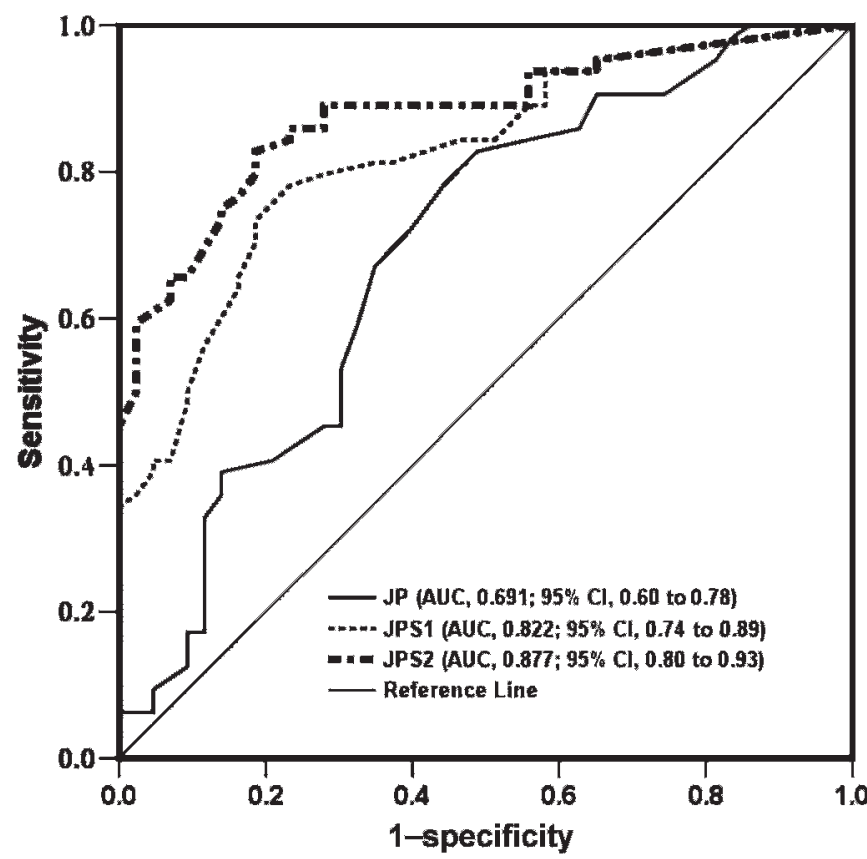

Figure 4 ROC curves for comparing MDCT-diagnostic performance in detecting reversible perfusion by SPECT-SRS $\geqslant 2$. JP extent of jeopardised myocardium by MDCT; JP1, JP multiplied by the weighted factor of CTA-culprit lesion ( $<40 \%, 0 ; 40-70 \%, 0.5 ;>70 \%$, 1); JP2, JP multiplied by the weighted factor of CTA-culprit lesion $(<40 \%, 0 ; 40$ $70 \%, 0.5 ;>70-90 \%, 1 ;>90-100 \%$ with distal contrast filling, 2 ; and $100 \%$ without distal contrast filling, 1). (AUC: JP2 vs JP, $p<0.001$; JP2 vs JP1, $\mathrm{p}=0.007$; JP1 vs JP, $\mathrm{p}<0.001$ ).

demonstrated that in fusion images using CCA with SPECT, the anatomical mass at risk from CCA and physiological mass at risk from SPECT correlated well $(\mathrm{r}=0.76){ }^{23}$ In the present study, correlation between MDCT-jeopardised score and SPECT-SRS was 0.741. The reasons for variations between scores may include the following. (1) By assigning arbitrary weighting factors to residual stensois and presence or absence of contrast filling distal to total occlusion, the haemodynamic effect may be oversimplified, especially the intermediate severity (40\% to $70 \%$ ). (2) Jeopardised myocardium detected by SPECT can be diminished by good collateral circulation, which provides myocardial protection, but increased if collateral

Table 3 Accuracy of MDCT according to the presence and absence of SPECT-SRS $\geqslant 2$ without/with corresponding significant stenoses on CCA in the infarct-related territory

\begin{tabular}{lll}
\hline & SPECT-SRS $\geqslant 2$ & $\begin{array}{l}\text { SPECT-SRS } \geqslant 2 \\
\text { plus CCA }\end{array}$ \\
\hline $\begin{array}{l}\text { CTA (culprit lesion 50\%) } \\
\quad \text { Sensitivity }\end{array}$ & $93.8 \%(60 / 64)$ & $98.4 \%(60 / 61)$ \\
$\quad$ Specificity & $18.6 \%(8 / 43)$ & $23.9 \%(11 / 46)$ \\
$\quad$ Negative predictive value & $66.7 \%(8 / 12)$ & $91.7 \%(11 / 12)$ \\
Positive predictive value & $63.2 \%(60 / 95)$ & $63.2 \%(60 / 95)$ \\
$\quad$ Accuracy & $63.6 \%(68 / 107)$ & $68.2 \%(71 / 107)$ \\
MDCT-jeopardised score $(2.5)$ plus & & \\
CTA (culprit lesion 50\%) & & \\
Sensitivity & $85.9 \%(55 / 64)$ & $90.2 \%(55 / 61)$ \\
Specificity & $79.1 \%(34 / 43)$ & $80.4 \%(37 / 46)$ \\
$\quad$ Negative predictive value & $79.1 \%(34 / 43)$ & $86.0 \%(37 / 43)$ \\
Positive predictive value & $85.9 \%(55 / 64)$ & $85.9 \%(55 / 64)$ \\
Accuracy & $83.2 \%(89 / 107)$ & $86.0 \%(92 / 107)$ \\
\hline
\end{tabular}

arteries have significant lesions. ${ }^{24}$ The area is also influenced by the presence of co-dominance or multiple diseased vessels due to the balanced ischaemic effect. (3) Comparing segments by two different modalities could have some degree of misclassification and account for the imperfect association between anatomically based and physiologically based risk areas.

The functional impact of reversibility is a continuous range, but clinical decision-making is binary in distinguishing reversible from non-reversible infarct-related myocardium. In daily practice, when SPECT-SRS $\geqslant 2$, CCA adds luminology information to aid planning the treatment. Like previous studies of culprit lesions evaluated by CTA alone, ${ }^{25} 26$ the diagnostic performance for IRA revascularisation is limited (table 3). However, if the extent of jeopardised myocardium and culprit lesion severity are evaluated together, the accuracy is good $(86.0 \%)$. These findings suggest that dual-phase MDCT is wellsuited for assessing clinical relevance post-AMI.

The drawback of radiation exposure should be considered for patients undergoing the dual-phase MDCT protocol, even though ECG-controlled tube current modulation was adopted. The estimated mean effective radiation dose in the MDCT protocol was about $14-20 \mathrm{mSv}$; this is not higher than that administered by Tl-201 (around $17 \mathrm{mSv}$ ) plus CCA (around 4$6 \mathrm{mSv}$ ). ${ }^{27}$ The study also provides a starting point for future investigations with new scanners or techniques such as low radiation exposure using prospectively ECG triggering. ${ }^{29}$

\section{Limitations}

First, only uncomplicated AMI patients were included. Second, the additional injection of T1-201 was not administered when redistribution images were obtained owing to consideration of radiation exposure. This may reduce the sensitivity in detecting reversible segments. Third, we included a relatively small group. Future studies with larger populations are needed to confirm our results. Fourth, an absolute quantitative approach was not used to determine the extent of jeopardised/infarcted myocardium. Currently, the resolution of epicoronary diameter less than 1.0 $\mathrm{mm}$ and intra-myocardial vessel detection by $3 \mathrm{D}$ volume-rendered image are still limited. Therefore, it was semi-quantified by the 17-segment model, which is widely accepted in daily practice. Finally, six patients were excluded because of motion artefacts or severe calcification at the culprit lesion/non-culprit lesion segments, interfering with the accuracy of measurement. The latest generation MDCT (such as advanced dual-source MDCT) may be more suitable for these patients after AMI.

\section{CONCLUSIONS}

The combination of dual-phase MDCT myocardial maps plus CTA enables a non-invasive strategy for identifying IRA, assessing extent of infarcted and jeopardised myocardium and determining IRA revascularisation. It can provide an alternative choice for risk triage and therapeutic planning in selected patients after AMI (3-7 days).

Funding: Supported by National Science Council, Taiwan, NSC 97-2314-B-010-045MY3 and Kaohsiung Veterans General Hospital, Taiwan, grant no VGHKS97-007.

Competing interests: None.

Provenance and peer review: Not commissioned; externally peer reviewed.

\section{REFERENCES}

1. Antman EM, Anbe DT, Armstrong PW, et al. 2007 Focused update of the ACC/AHA 2004 guidelines for the management of patients with ST-elevation myocardial infarction. A report of the American College of Cardiology/American Heart Association Task Force on practice guidelines. Circulation 2007;116:e148-304. 
2. Anderson JL, Adams CD, Antman EM, et al. ACC/AHA 2007 guidelines for the management of patients with unstable angina/non-ST-elevation myocardial infarction: a report of the American College of Cardiology/American Heart Association Task Force on Practice Guidelines (Writing Committee to Revise the 2002 Guidelines for the Management of Patients With Unstable Angina/Non-ST-Elevation Myocardial Infarction). J Am Coll Cardiol 2007:50:e1-e157.

3. Mollet NR, Cademartiri F, van Mieghem CA, et al. High-resolution spiral computed tomography coronary angiography in patients referred for diagnostic conventional coronary angiography. Circulation 2005;112:2318-23.

4. Raff GL, Gallagher MJ, O'Neill WW, et al. Diagnostic accuracy of noninvasive coronary angiography using 64-slice spiral computed tomography. J Am Coll Cardiol 2005; $46: 552-7$

5. Meijboom WB, Mollet NR, Van Mieghem CA, et al. 64-Slice CT coronary angiography in patients with non-ST elevation acute coronary syndrome. Heart 2007:93:1386-92.

6. Abbara S, Chow BJ, Pena AJ, et al. Assessment of left ventricular function with 16and 64-slice multi-detector computed tomography. Eur J Radiol 2008;67:481-6.

7. Gerber BL, Belge B, Legros GJ, et al. Characterization of acute and chronic myocardial infarcts by multidetector computed tomography: comparison with contrast-enhanced magnetic resonance. Circulation 2006;113:823-33.

8. Lardo AC, Cordeiro MA, Silva C, et al. Contrast-enhanced multidetector computed tomography viability imaging after myocardial infarction: characterization of myocyte death, microvascular obstruction, and chronic scar. Circulation 2006;113:394-404.

9. Lessick J, Dragu R, Mutlak D, et al. Is functional improvement after myocardial infarction predicted with myocardial enhancement patterns at multidetector CT? Radiology 2007:244:736-44.

10. Sato A, Hiroe $\mathrm{M}$, Nozato $\mathrm{T}$, et al. Early validation study of 64-slice multidetector computed tomography for the assessment of myocardial viability and the prediction of left ventricular remodelling after acute myocardial infarction. Eur Heart $J$ 2008;29:490-8.

11. Chiou KR, Liu CP, Peng NJ, et al. Identification and viability assessment of infarcted myocardium with late enhancement multidetector computed tomography: comparison with thallium single photon emission computed tomography and echocardiography. Am Heart J 2008;155:738-45.

12. Einstein AJ, Moser KW, Thompson RC, et al. Radiation dose to patients from cardiac diagnostic imaging. Circulation 2007;116:1290-305.

13. Cerqueria MD, Weissman NJ, Dilsizian V, et al. Standardized myocardial segmentation and nomenclature for tomographic imaging of the heart: a statement for healthcare professionals from the Cardiac Imaging Committee of the Council on Clinical Cardiology of the American Heart Association. Circulation 2002;105:539-42.

14. Ilia R, Rosenshtein G, Weinstein J, et al. Left anterior descending artery length in left and right coronary artery dominance. Coron Artery Dis 2001;12:77-8.
15. Huang WC, Wu MT, Chiou KR, et al. Assessing culprit lesions and active complex lesions in patients with early acute myocardial infarction by multidetector computed tomography. Circ J 2008;72:1806-13.

16. Candell-Riera J, Santana-Boado C, Castell-Conesa J, et al. Culprit lesion and jeopardized myocardium: correlation between coronary angiography and singlephoton emission computed tomography. Clin Cardio 1997;20:345-50.

17. Pereztol-Valdés 0, Candell-Riera J, Santana-Boado C, et al. Correspondence between left ventricular 17 myocardial segments and coronary arteries. Eur Heart $J$ 2005:26:2637-43

18. Schuijf JD, Wijns W, Jukema JW, et al. A comparative regional analysis of coronary atherosclerosis and calcium score on multislice CT versus myocardial perfusion on SPECT. J Nucl Med 2006;47:1749-55.

19. Schuijf JD, Wijns W, Jukema JW, et al. Relationship between noninvasive coronary angiography with multi-slice computed tomography and myocardial perfusion imaging. J Am Coll Cardiol 2006;48:2508-14.

20. Goldstein JA, Demetriou D, Grines $\mathrm{CL}$, et al. Multiple complex coronary plaques in patients with acute myocardial infarction. N Engl J Med 2000;343:915-22.

21. Seiler C, Kirkeeide RL, Gould KL. Measurement from arteriograms of regional myocardial bed size distal to any point in the coronary vascular tree for assessing anatomic area at risk. J Am Coll Cardiol 1993;21:783-97.

22. Graham MM, Faris PD, Ghali WA, et al. Validation of three myocardial jeopardy scores in a population-based cardiac catheterization cohort. Am Heart $J$ 2001;142:254-61.

23. Faber TL, Santana CA, Garcia EV, et al. Three-dimensional fusion of coronary arteries with myocardial perfusion distributions: clinical validation. J Nucl Med 2004:45:745-53.

24. Nohra R, Kambara H, Murakami T, et al. Collateral function in early acute myocardial infarction. Am J Cardiol 1983;52:955-9.

25. Gaemperli 0, Schepis T, Koepfli P, et al. Accuracy of 64-slice CT angiography for the detection of functionally relevant coronary stenoses as assessed with myocardial perfusion SPECT. Eur J Nucl Med Mol Imaging 2007;34:1162-171.

26. Hacker M, Jakobs T, Matthiesen $\mathrm{F}$, et al. Comparison of spiral multidetector CT angiography and myocardial perfusion imaging in the noninvasive detection of functionally relevant coronary artery lesions: first clinical experiences. J Nucl Med 2005:46:1294-300

27. Einstein AJ. Radiation risk from coronary artery disease imaging: how do different diagnostic tests compare? Heart 2008;94:1519-21.

28. Coles DR, Smail MA, Negus IS, et al. Comparison of radiation doses from multislice computed tomography coronary angiography and conventional diagnostic angiography. J Am Coll Cardiol 2006;47:1840-5.

29. Earls JP, Berman EL, Urban BA, et al. Prospectively gated transverse coronary CT angiography versus retrospectively gated helical technique: improved image quality and reduced radiation dose. Radiology 2008;246:742-53. 